\section{Comparison of the hormonal responses to exhaustive incremental exercise in adolescent and young adult males}

\author{
Comparação das respostas hormonais ao exercício incremental até \\ exaustão em adolescentes e adultos jovens do sexo masculino
}

Anthony C. Hackney', Mehis Viru², Mitch VanBruggen', Tamara Janson ${ }^{3}$, Kalle Karelson ${ }^{3}$, Atko Viru ${ }^{3}$
1 Endocrine Section - Applied Physiology Laboratory, University of North Carolina, Chapel Hill, NC, USA ${ }^{2}$ Institute of Sport Pedagogy and Coaching Science, University of Tartu, Tartu, Estonia

${ }^{3}$ Institute of Exercise Biology and Physiotherapy, University of Tartu, Tartu, Estonia
Correspondence to: Anthony C. Hackney University of North Carolina CB \# 8700, Fetzer Hall, Chapel Hill, 27599 - North Carolina, United States

ach@email.unc.edu

Received on Nov/21/2010 Accepted on Feb/16/2011 dos no EX ocorreram no cortisol ( $p<0,01$, aumentou com a elevação do estágio $E X)$ e no de testosterona ( $p<0,01 ; \mathrm{TS} 4<\mathrm{TS5}$, adultos). Houve efeito de interação entre grupos e por estágio de EX no grupo de hormônio de crescimento $(p<0,05)$. O hormônio de crescimento aumentou em todos os grupos em resposta ao EX, no entanto, a magnitude dos aumentos observados foi significativamente menor em TS5 e nos adultos do que em TS4. Conclusões: As diferenças verificadas nas respostas dos grupos de testosterona e hormônio de crescimento em TS4 em relação aoTS5 e adultos refletem os diferentes níveis de maturação do sistema endócrino entre os estágios do índice de Tanner. Os adolescentes noTS5 se assimilam mais aos jovens adultos do que aos adolescentes TS4, quanto a suas respostas hormonais ao EX.Arq Bras Endocrinol Metab. 2011;55(3):213-8

Descritores

Atividade física; sistema endócrino; Etapa Tanner; jovem

\section{INTRODUCTION}

$\mathrm{I}$ $\mathrm{t}$ is well established that physical exercise acts as a stress to the endocrine system (1). Particularly with maximal levels of physical exercise, such as in sporting activities, dramatic changes in circulating hormonal concentrations can occur $(1,2)$. The number of research 
studies examining the endocrine response to exercise has significantly increased over the last few decades as analytical methods have become more refined, available, and affordable to researchers. These studies have carefully characterized and profiled the hormonal behavior for exercise of different intensities, durations, modalities, and environmental settings. However, most of these studies have focused on examining the exercise hormonal responses of adults and not those of children and adolescents.

Many leading scientific agencies, such as the World Health Organization (WHO), acknowledge that there is a public health crisis relative to the increasing prevalence of obesity occurring worldwide (3). In particular, there is alarm concerning the rate of obesity development in children and adolescents; in fact, they are the most rapidly expanding segment of the population entering the obese ranks (3). Exercise science, nutrition and public health researchers all recognize that one of the key causative aspects to the developing obesity problem is an increase in sedentary lifestyle (i.e., which can lead to excessive positive energy balance). This has resulted in such researchers calling for increased levels of physical exercise in children and adolescents (3). However, the physiological responses to exercise in the pediatric population are not clearly understood, fostering the need for more research to characterize such responses (4). This point is especially true and critical to the endocrine system, which through its hormonal mediator system regulates many aspects of metabolism and energy balance at rest and during exercise.

Therefore, with the above points in mind the present investigation was undertaken. The study purpose was to characterize select hormonal responses to incremental exercise of maximal intensity levels (i.e., to exhaustion) in male adolescents at different Tanner Stages of development in comparison to those of young adult males.

\section{MATERIAL AND METHODS}

\section{Subjects}

In accordance with the Helsinki Declaration, written informed consent was obtained from all subjects (males, 6 adolescents and 4 young adults) after aspects of the study had been explained to them. The subjects ranged in age from 16 to 21 and were experienced with performing research testing as well as in regular sports training ( $\geq 3$ years of primarily aerobic exercise activities; e.g., running, cross-country skiing, swimming). None of the subjects suffered from any chronic or acute diseases or health problems, they were all non-smokers and did not consume alcohol or take any prescription medications. For this study the adolescents were tested twice (in Tanner Stage 4 [TS4, 16 years old] and Tanner Stage 5 [TS5, 17 years old] separated by approximately a one year period [ \pm one week]) and the adults $(21 \pm 1$ years old; $\mathrm{X} \pm \mathrm{SD})$ were tested once. Tanner Stage status was based upon medical criteria, questionnaire, and hormonal status (see Analytical Methods section) $(5,6)$. The physical characteristics of the subjects appear in Table 1 .

Table 1. Physical characteristics of the subjects and select physiological responses to the maximal exercise test in the Tanner Stage 4 (TS4) and Tanner Stage 5 (TS5) adolescents and young adult males ( $21 \pm 1$ years old). All values are means $\pm S D$

\begin{tabular}{lccc}
\hline & TS4 (16y) & TS5 (17y) & Adults \\
\hline Height $(\mathrm{cm})$ & $183 \pm 7$ & $185 \pm 6^{*}$ & $177 \pm 6^{\star \star}$ \\
Mass $(\mathrm{kg})$ & $72.2 \pm 7.4$ & $75.3 \pm 7.2^{*}$ & $79.5 \pm 12.9$ \\
Body mass index $\left(\mathrm{kg} \cdot \mathrm{m}^{-2}\right)$ & $21.5 \pm 1.5$ & $21.9 \pm 1.6$ & $25.4 \pm 4.9^{\star \star}$ \\
Peak V0 $\left(\mathrm{mL} . \mathrm{kg}^{-1} \cdot \mathrm{min}^{-1}\right)$ & $44.5 \pm 7.4$ & $52.4 \pm 7.8$ & $53.1 \pm 5.2$ \\
$\%_{\text {VO }}$ at lactate threshold & $76.8 \pm 9.8$ & $74.1 \pm 10.8$ & $74.4 \pm 8.7$ \\
Peak lactate $\left(\mathrm{mmol} . \mathrm{L}^{-1}\right)$ & $9.8 \pm 3.2$ & $10.2 \pm 1.1$ & $9.2 \pm 2.6$ \\
\hline
\end{tabular}

${ }^{*}$ Differs from TS4; ${ }^{\star *}$ Differs from TS4, TS5 $(\mathrm{p}<0.05)$.

\section{Procedures}

Every effort was made to standardize and control the settings for the exercise testing of the subjects. The exercise tests were performed between 01:00 and 03:00 p.m. each day. To ensure stable blood glucose levels, the subjects were asked not to eat for the 3 hours before the exercise testing and to make their last meal small in size $(\sim 300 \mathrm{kcal})$. The subjects were also asked to avoid strenuous physical exertion or psychological strain during the 36 hours prior to all exercise testing. As noted, all subjects were experienced in exercise research participation, which minimized any potential anticipatory - anxiety responses in their physiological outcomes.

Upon reporting to the laboratory, each subject rested (seated position) for 30 minutes, and then a sterile IV catheter was inserted into an antecubital vein. After an additional 30 minute period, a resting blood sample was taken. Following a light warm-up $(\sim 5 \mathrm{mi}-$ nutes), cycle ergometry exercise began with an initial power output of 80 Watts $(\mathrm{W})$ for 4 minutes. The po- 
wer output was increased by $40 \mathrm{~W}$ every 4 minutes up to $240 \mathrm{~W}$ (fifth stage). The next and final stage of the incremental exercise consisted of a one-minute sprint at the highest possible rate of pedaling (i.e., simulating a competition scenario and to make the exercise effort maximal, exhaustive. A blood specimen was collected at the end of each exercise stage as well as at the very end of exercise. Blood specimens were collected into sterile vacutainer tubes, allowed to clot, and then immediately centrifuged at $4^{\circ} \mathrm{C}$ to separate serum. Serum specimens were refrigerated and stored at $-20^{\circ} \mathrm{C}$ until later biochemical analysis could be performed.

During the entire period of the incremental exercise test expired respiratory gases were collected and analyzed continuously, using a Parvo Medics TrueMax 2400 Metabolic Measurement System calorimetry system (Sandy, Utah, USA). The highest oxygen uptake obtained from the respiratory gases was considered to be the peak oxygen uptake for the exercise test (peak $\mathrm{VO}_{2}$ ). Throughout exercise testing strong verbal encouragement was given to the subjects.

\section{Analytical methods}

Hormonal concentrations for cortisol, total testosterone, and growth hormone $(\mathrm{GH})$ were determined in all serum specimens by a highly specific automated immuno-chemoluminescence system (7). Assay detection limit, intra-assay variation, and inter-assay variation were: cortisol $=5.5 \mathrm{nmol} \cdot \mathrm{L}^{-1}, 7.0 \%$ and $10.3 \%$; testosterone $=0.5 \mathrm{nmol} \cdot \mathrm{L}^{-1}, 6.7 \%$, and $7.7 \% ; \mathrm{GH}=0.7$ ng. $L^{-1}, 7.2 \%$ and $9.1 \%$, respectively. Blood lactate concentrations were also measured in serum specimens to determine lactate threshold point (exercise intensity) and peak lactate responses, using methods previously described elsewhere (8). All specimen handling and analytical procedures were standardized and based on recommendations present in the literature to ensure viable biochemical outcomes (9).

\section{Statistics}

A mixed-model (age group vs. measurement time) analysis of variance (ANOVA) was used in combination with post hoc testing (Bonferroni), where appropriate, to perform statistical analysis on mean responses of all measurements. For all statistical analyses the accepted probability level was designated as $\mathrm{p} \leq 0.05$. Additionally, for all significance between age group (TS4 vs. TS5 vs. adult) hormonal findings, effect size calculations
(Cohen's $d$ ) were conducted to determine the physiological meaningfulness of outcome differences (10).

\section{RESULTS}

\section{Physical characteristics - Exercise response}

Table 1 presents the physical characteristics and outcomes of exercise testing for all the subjects. Adolescents at TS5 were taller $(\mathrm{p}<0.05)$ than they had been at TS4, and were taller $(\mathrm{p}<0.05)$ than the adults in the study. Likewise, the adolescents increased in body mass from TS4 to TS5 ( $\mathrm{p}<0.05)$, but did not differ from adults at either Tanner Stage. The adults, however, did have a greater $(\mathrm{p}<0.05)$ body mass index $(\mathrm{BMI})$ than the adolescents at both TS4 and TS5.

Peak $\mathrm{VO}_{2}$ increased from TS4 to TS5 in the adolescents $(\mathrm{p}<0.05)$, and the adult group peak $\mathrm{VO}_{2}$ was greater $(\mathrm{p}<0.05)$ than that of the adolescents at TS4 but not at TS5. The percentage of peak $\mathrm{VO}_{2}$ that elicited the lactate threshold was similar in adolescents and adults regardless of the age group. Correspondingly, the peak lactate response to exercise did not vary between the subjects regardless of age group.

\section{Hormonal outcomes}

Cortisol responses of the adolescents at TS4 and TS5 as well as that of the adults progressively increased $(\mathrm{p}<$ 0.01 ) from rest (time 0 ) to the end of the exercise test at the final sprint $(\mathrm{sp})$ (Figure 1). However, no group differences occurred in the magnitude of responses between the adolescents (TS4 or TS5) and the adults. There was a slight tendency for cortisol levels of both the TS4 and TS5 adolescents to be elevated at rest in comparison to the young adults, but this effect only approaches statistical significance $(\mathrm{p}<0.10>0.05)$ (TS4+TS5 vs. adults; Cohen's $d=0.67$ ).

Figure 2 displays the testosterone responses of the subjects. There was a significant difference $(\mathrm{p}<0.05)$ between the age groups for this hormonal response. TS4 testosterone levels were significantly lower than those of the TS5 adolescents and adults across the entire exercise test sampling times (TS4 vs. TS5+adults; Cohen's $d=1.58$ ); however, none of the responses of the TS5 and adults differed from one another. Furthermore, progression of exercise intensity through the various testing stages (rest to exhaustion) did not significantly affect the level of testosterone in the TS4, TS5 or adult age groups. 


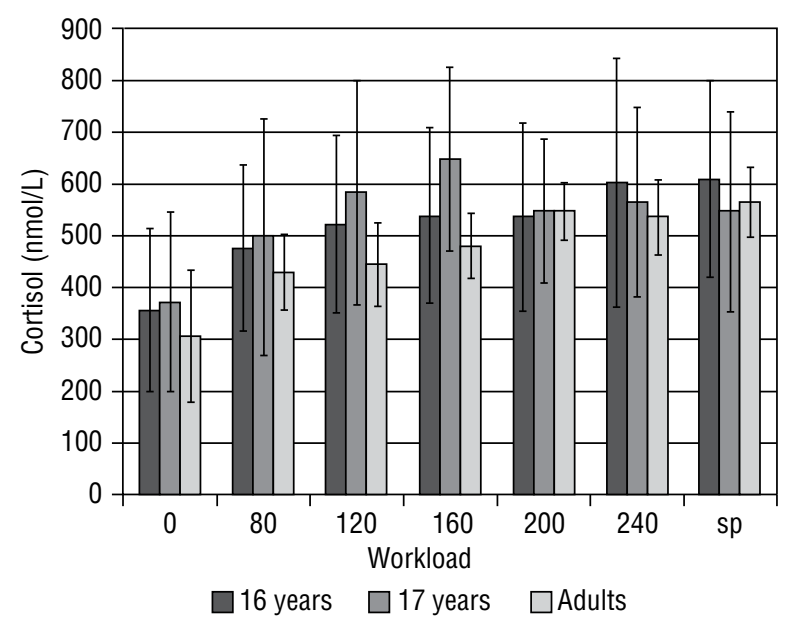

Figure 1. Cortisol responses $(X \pm S D)$ of adolescent subjects in Tanner Stage 4 (16 years old), in Tanner Stage 5 (17 years old), and in a group of young adults (21 years old). The y-axis in the figure represents the hormonal concentration in the blood of the subjects, while the $x$-axis is the resistance workload (i.e., power) in Watts $(W)$ during the incremental cycling exercise test. The abbreviation "sp" denotes the sprint stage until exhaustion, at the end of exercise.

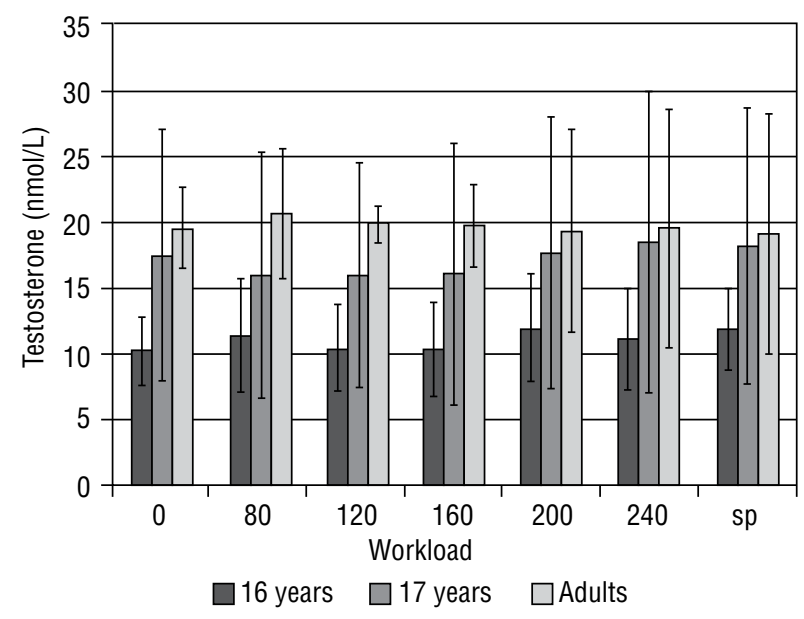

Figure 2. Testosterone responses $(X \pm S D)$ of the adolescent subjects in Tanner Stage 4 (16 years old), in Tanner Stage 5 (17 years old), and in a group of young adults (21 years old). The $y$-axis in the figure represents the hormonal concentration in the blood of the subjects, while the $x$-axis is the resistance workload (i.e., power) in Watts (W) during the incremental cycling exercise test. The abbreviation "sp" denotes the sprint stage until exhaustion at the end of exercise.

$\mathrm{GH}$ responses are shown in figure 3. The resting and exercise GH levels of the TS4 adolescents tended to be greater than those of both the TS5 adolescents and adults. However, due to a large amount of variability within the $\mathrm{GH}$ responses in all age groups, these between group differences only approached statistical significance $(\mathrm{p}=0.10)$. Exercise did result, however, in significant increases $(\mathrm{p}<0.05)$ in $\mathrm{GH}$ from rest (time 0$)$ to the final sprint (sp) at the end of the exercise test for all age groups. Furthermore, peak GH (time sp) of the TS4 adolescents was greater $(\mathrm{p}<0.05)$ than that of the TS5 adolescents and adults (TS4 vs. TS5+adults; Cohen's $d=1.86$ ); although, the latter two age groups did not differ from one another in peak GH responses.

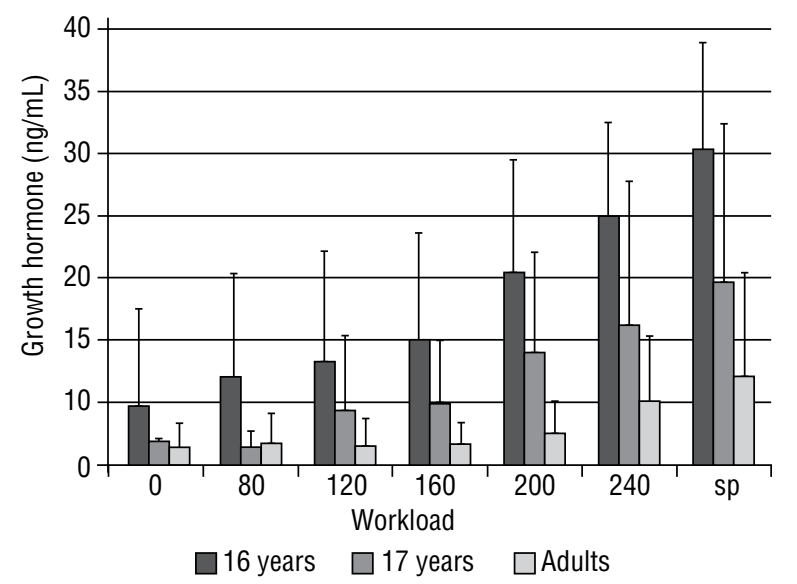

Figure 3. Growth hormone responses $(X \pm S D)$ of the adolescent subjects in Tanner Stage 4 ( 16 years old), in Tanner Stage 5 (17 years old), and in a group of young adults (21 years old). The $y$-axis in the figure represents the hormonal concentration in the blood of the subjects, while the x-axis is the resistance workload (i.e., power) in Watts (W) during the incremental cycling exercise test. The abbreviation "sp" denotes the sprint stage until exhaustion at the end of exercise.

\section{DISCUSSION}

Physical inactivity has been linked to the epidemic of obesity occurring worldwide. Regrettably, children and adolescents are part of the fastest growing segment of the population subsuming to this epidemic. This latter occurrence has resulted in a need for more research on the responses of children and adolescents to exercise as a means to better understand their unique physiology. Characterizing hormonal responses to exercise are a fundamental part to this understanding due to the endocrine system's multifaceted role in overall metabolic regulation. Thus, the aim of this study was to examine the cortisol, testosterone and $\mathrm{GH}$ responses to exercise in adolescents at different developmental ages in comparison to that of young adults. In general, the results suggest adrenal cortisol response to exercise is very similar between adults and adolescents in Tanner Stage 4 and 5 . However, gonadal testosterone response to exercise is lower in Tanner Stage 4 than that of Tanner Stage 5 adolescents or in adults. Furthermore, the ante- 
rior pituitary GH response to exercise of Tanner Stage 4 adolescents is much greater than that of Tanner Stage 5 or of young adults.

On the whole, the hormonal findings for the adolescent groups in this study are in agreement with previously published reports. For example, Viru and cols. showed that exercise induces increased cortisol responses in adolescents and young adults $(2,11,12)$. These investigators also found a lowering of cortisol levels at rest and with exercise as adolescents move from Tanner Stage 2 to Stage 5 (12). Boisseau and Delamarche and Wirth and cols. also reported similar occurrences $(4,13)$. The adolescents in the present study, however, only tended to display this latter response; i.e., the data approached statistical significance. Kraemer and others reported equivalent cortisol responses in adolescents completing a very similar exercise protocol; although they studied only young girls (14). Relative to testosterone, Fahey and cols. reported nearly identical findings as the present study for this hormone, both at rest and in response to exercise in Tanner Stages 4 and 5 adolescents (15). Likewise, the present GH findings, at rest and in response to exercise, also matched those reported by Fahey and associates in adolescents (15). Furthermore, the work of Veldhuis and colleagues has shown there is a strong influence of age on the circulating levels of $\mathrm{GH}$ and $\mathrm{GH}$ production at the anterior pituitary in young versus older adults (i.e., decreasing with advancing age) (16). The present data support such phenomena occurring even when progressing from Tanner Stage 4 to young adulthood.

While our data replicates and support previous research findings, there is a unique aspect to our study. The hormonal profiling used involved a frequent blood sampling protocol during exercise to compare age groups. Many previous studies simply used a pre-post exercise blood sampling protocol approach. The present blood sampling protocol allows for a more dynamic characterization of the hormonal responses to exercise. This characterization revealed that the differences observed for testosterone and $\mathrm{GH}$ in the adolescents seem to emanate from the hormonal differences observed at rest prior to exercise, and are not due to differing response patterns during the exercise. Interestingly, the similarity of the exercise responses, once resting-basal differences are accounted for, suggest that the regulatory aspects of the endocrine system are responsive to the stimulus of physical exercise in a very similar fashion in males at Tanner Stage 4, 5, and in young adults. Thus, for corti- sol, testosterone and GH the endocrine glands producing these hormones are responsive to physical exercise as a stressful stimulus but relative to testosterone and GH the overall amount of hormone being produced is at variance with that of young adults ( $\downarrow$ testosterone, $\uparrow \mathrm{GH}$ ). To our knowledge this finding has not been thoroughly reported previously in the literature.

The testosterone and GH differences observed at rest and throughout exercise in the Tanner Stage 4 adolescents most likely reflect the less mature developmental levels of the endocrine system within that age group $(5,6,17)$. As would be expected, the Tanner Stage 5 adolescents are much more similar in their responses to that of young adults than the Tanner Stage 4 adolescents. This suggests that at this age level (16-17 years) a one-year period of time is critical to developmental progression not only of the endocrine system but also of the skeletal muscle and cardiopulmonary systems (as implied by the observed changes in body mass as well as peak $\mathrm{VO}_{2}$ ) (18).

The interpretation of our data is limited by the small sample size we employed in the study. This most certainly added to the variability encountered in some of the observed hormonal outcomes. We acknowledge this constraint and therefore suggest that care be used in the application and use of our findings. However, for our between group differences we calculated effect size statistics, and all of our significant findings had "large" effect sizes (19). These substantially large effect sizes support the physiological importance and meaningfulness of our findings and offset our small sample size. Furthermore, this study used extensive systematic controls and rigid standardization procedures throughout to ensure that the data gathered were highly valid and reliable, thus adding to the strength of the research study. Additionally, our unique blood sampling protocol allowed us to examine hormonal responses through the various stages of our incremental increases in exercise intensity. To our knowledge, this has not been done before (most exercise studies simply examine hormonal response immediately before and after exercise). Collectively, these points support the scientific validity of our findings.

In conclusion, Tanner Stage 4 male adolescents do have differing hormonal response to exercise than Tanner Stage 5 adolescents or young adults. However, these differences seem to be due to pre-existing resting-basal level variances that are reflective of the diverse maturation states within the endocrine system. It is ad- 
vised that exercise researchers who study young athletes at or across these two different Tanner Stages account for the pre-existing resting hormonal differences; and thus, treat such adolescents as two distinctly different subject groups. To not do so could result in confounding effects in hormonal research outcomes and invalidate data interpretation and understanding.

Acknowledgements: This study was funded by the Estonian Scientific Foundation, Grant 3962.

Disclosure: no potential conflict of interest relevant to this article was reported.

\section{REFERENCES}

1. Hackney AC. Stress and the neuroendocrine system: the role of exercise as a stressor and modifier of stress. Expert Rev Endocrinol Metab. 2006;1(6):783-92.

2. Viru A. Plasma hormones and physical exercise. Int J Sports Med. 1992;13:201-9.

3. Population-based Prevention Strategies for Childhood Obesity. 2010. The World Health Organization. p. 3-37.

4. Boisseau N, Delamarche P. Metabolic and hormonal responses to exercise in children and adolescents. Sports Med. 2000;30(6):405-22.

5. August GP, Grumbach MM, Kaplan S. Hormonal changes in puberty. III Correlation of plasma testosterone, LH, FSH, testicular size and bone age with male pubertal development. J Clin Endocrinol Metab. 1972;34:319-26.

6. Preece MA. Prepubertal and pubertal endocrinology. In: Falkner $F$, Tanner JM, eds. Human growth: a comprehensive treatise. Postnatal growth Neurobiology. 2nd edition. NewYork: Plenum, 1986, vol. 2, p. 211-24.
7. Babson A. The IMMULITE. Automated immunoassay system. J Clin Immunoassay. 1991;14:83-8.

8. Port K. Serum and saliva cortisol response and blood lactate accumulation during incremental exercise testing. Int J Sports Med. 1992;12:490-4.

9. Hackney AC, Viru A. Research methodology: endocrinologic measurements in exercise science and sports medicine. J Athl Train. 2008;43(6):631-9.

10. Cohen J. Statistical power analysis for the behavioral sciences. 2nd edition. Erlbaum, Hillsdale, NJ, 1988.

11. Viru A, Laaneots L, Karelson K, Smirnova T, Viru M. Exercise-induced hormone responses in girls at different stages of sexual maturation. Eur J Appl Physiol. 1998;77:401-8.

12. Viru A, Karelson K, Smirnova T. Stability and variability in hormone responses to prolonged exercise. Int $\mathrm{J}$ Sports Med. 1992;13:230-5.

13. Wirth A, Träger E, Scheele K, Mayer D, Diehm K, Reischle K, et al. Cardiopulmonary adjustment and metabolic response to maxi$\mathrm{mal}$ and submaximal physical exercise of boys and girls at different stages of maturity. Eur J Appl Physiol. 1978;39:229-40.

14. Kraemer RR, Acevedo EO, Synovitz LB, Hebert EP, GimpelT, Castracane VD. Leptin and steroid hormone responses to exercise in adolescent female runners over a 7-week season. Eur J Applied Physiol. 2001;86:85-91.

15. FaheyTD, Valle-Zuris AD, Oehlsen G, Trieb M, Seymour J. Pubertal stage differences in hormonal and hematological responses to maximal exercises in males. J Appl Physiol. 1979;46:823-7.

16. Veldhuis JD, Weltman JY, Weltman A, Iranmanesh A, Muller E, Bowers $\mathrm{CY}$. Age and secrtagogue type jointly determine dynamic growth hormone responses to exogenous insulin-like growth factor-negative feedback in healthy men. J Clin Endocrinol Metab. 2004;89(11):5542-8.

17. Grumbach MM. The neuroendocrinology of human puberty revisited. Horm Res. 2002;57 Suppl. 2:2-14.

18. Baxter-Jones A, Goldstein H, Helms P. The development of aerobic power in young athletes. J Appl Physiol. 1993;74:1160-97.

19. Ferguson CJ. An effect size primer: a guide for clinicians and researchers. Prof Psych Res Prac. 2009;40(5):532-8. 